\title{
Parameter Estimation of an Electrochemical Supercapacitor Model
}

\author{
Ross Drummond, David A. Howey, Stephen R. Duncan
}

\begin{abstract}
Parameter estimation of a supercapacitor model that uses PDEs to describe electrochemical physical phenomena is studied. The model parameters were identified from time domain charge/discharge data and also from frequency domain data in the form of electrical impedance spectroscopy at different open cell voltages. The identified physical model could fairly accurately simulate the experimental data, however, this accuracy was found to drop off at high frequencies. The performance of the physical model was compared to a second order circuit, with the two models having similar levels of accuracy. The circuit was found to be simpler to implement but, unlike the physical model, could not supply physical information about the internal state of the supercapacitor.
\end{abstract}

\section{INTRODUCTION}

This paper is concerned with using experimental data to estimate the parameters of a supercapacitor model that describes electrochemical physical phenomena. Supercapacitors are electrical energy storage devices that are characterised by low internal resistances, high cyclic efficiencies, reduced ageing and weaker dependencies upon temperature effects compared to other devices such as lithium ion batteries [12]. When supplied with power, a supercapacitor stores charge across an electric double layer which is a charge separating layer at the interface between the electrode and electrolyte. Compared to traditional capacitors, supercapacitors have significantly increased energy densities due to the increased surface areas of their porous electrodes and because the charge separating distance across the double layer is molecular in scale [12]. This unique set of energy storage characteristics has resulted in supercapacitors being successfully applied both in high power applications and also within hybrid power sources to complement the performance of other energy storage devices, such as fuel cells and lithium ion batteries.

Advances in electrochemistry have led to a significant improvement of electrochemical energy storage devices in recent years, leading to widespread adoption of the technology in areas such as grid storage, hybrid electric

Ross Drummond and Stephen R. Duncan are with the Control Group and David A. Howey is with the Energy and Power Group, Department of Engineering Science, University of Oxford, 17 Parks Road, OX1 3PJ Oxford, United Kingdom ross.drummond@eng.ox.ac.uk. Work supported in part by EPSRC grant EP/J010537/1. vehicles $(\mathrm{HEVs})$ and personal electronics. This popularity has led to increased demands on performance at a systems level. To provide this, batteries are normally implemented along with a battery management system (BMS) which makes predictions about the state of the device and subsequently computes input charging profiles [4]. For effective implementation, some form of management system should also be implemented for supercapacitors. One of the key functions of a BMS is to estimate how much remaining power can be drawn from the device. This is a key property for applications such as HEVs where "range anxiety", the driver's fear that they will run out of energy and not be able to rapidly recharge, is seen as one of the main reasons hindering the adoption of this technology [9]. In order for the BMS to make improved predictions, improved models need to be developed.

A number of supercapacitor models have already been proposed in the literature. These models can be considered as either atomistic models, equivalent circuits (ECs) or physics based (PB) models. Atomistic models are very detailed models that describe ionic interaction in complex spaces such as in the electrode pores [10],but they are not yet scalable and hence are not suitable for an online implementation within a BMS. ECs are the most popular form of supercapacitor model and have seen widespread adoption $[14,17]$. These models are relatively simple, both in terms of their theory and their computational implementation. The main disadvantage of circuit models is that they have no clear physical interpretation, so little additional information about the system can be estimated apart from the current/voltage response. Also, while circuits have been used to model system dynamics for decades, for example with the ideas of passive circuit synthesis [3], they are only 'local' models and may not be applicable for the whole charging profile.

PB models describe the transport of ions and evolution of the potential distribution during a charge using a set of conservation and diffusion relationships described by partial differential equations (PDEs). Solving these models is more complex than solving the linear ordinary differential equations (ODEs) of ECs, but, PB models are generally more powerful, giving a description of the internal state of the device. These models are not as well understood as ECs, but it is hoped that the increased information that PB models provide will enable improved designs of supercapacitors on a systems level, as they can establish links between changes in the physical parame- 


\begin{tabular}{c|c|c} 
& Definition & $\mathrm{Units}$ \\
\hline$\phi_{1}$ & Electrical potential in the electrode & $\mathrm{V}$ \\
$\phi_{2}$ & Electrical potential in the electrolyte & $\mathrm{V}$ \\
$c$ & Homogenised ionic concentration & $\mathrm{mol} \mathrm{m}^{-3}$ \\
$a C$ & Capacitance density & $\mathrm{F} \mathrm{m}^{-2}$ \\
$\sigma$ & Electrode conductivity & $\mathrm{S} \mathrm{m}^{-1}$ \\
$\epsilon$ & Porosity factor & \\
$D$ & Diffusion coefficient & $\mathrm{m}^{2} \mathrm{~s}^{-1}$ \\
$F$ & Faradays constant & $\mathrm{C} \mathrm{mol}^{-1}$ \\
$t_{+} /-$ & Relative mobility of $+/$- ions & \\
$d q_{+} /-/ d q$ & Change in ion surface concentration & \\
$\kappa$ & with electrode surface charge & \\
$R$ & Electrolyte conductivity & $\mathrm{S} \mathrm{m}^{-1}$ \\
$T$ & Universal gas constant & $\mathrm{J} \mathrm{K}^{-1} \mathrm{~mol}^{-1}$ \\
$c_{0}$ & Temperature & $\mathrm{K}$ \\
$S_{A}$ & Equilibrium concentration & $\mathrm{mol} \mathrm{m}^{-3}$ \\
$I$ & Surface area & $\mathrm{m}^{2}$ \\
$i / S A$ & Current & $\mathrm{A}$ \\
$V$ & Current density & $\mathrm{A} \mathrm{m}$ \\
$L$ & Voltage & $\mathrm{V}$ \\
& Length & $\mathrm{m}^{-2}$
\end{tabular}

TABLE I: Definitions of the PB model symbols.

ters and the electrical performance. It is also hoped that PB models will be more "global", being applicable for a wider domain of the state-space and not requiring online parameter estimation.

A benchmark PB model was introduced in [15] which discussed the electrochemical justifications and assumptions of the model PDEs and also compares its simulated response against constant-current charging profiles. An efficient computational implementation of this model was discussed in [8] and 3D effects of the model and some basic sensitivity analysis were carried out in [1]. Additional models have also been developed, for example that include temperature effects [5]. Aspects such as the observability of the model from [15] have also recently been studied [7]. Similar models have been developed for other types of energy storage devices, such as lithium ion batteries [6], however, these models are highly nonlinear and also more computationally intensive than equivalent physical supercapacitor models [2].

The purpose of this paper is to improve the accuracy of PB supercapacitor models by obtaining estimates of the model parameters that have physical interpretations from experimental data. Parameter estimation has been widely studied for circuits, for example [18], but it remains a somewhat open question for PB models. Experimental data in both the frequency and time domains of a commercially available Maxwell supercapacitor were used to estimate the parameters. The performance of the estimated $\mathrm{PB}$ model is compared against a $2^{\text {nd }}$ order circuit from [17].

This paper is structured as follows. Section 2 introduces the $\mathrm{PB}$ and $\mathrm{EC}$ models whose parameters are estimated. Section 3 discusses the experimental setup and the parameter estimation is studied in Section 4. Section 5 concludes the paper.

\section{MODELS}

The typical construction of a supercapacitor is similar to that of a battery having two electrodes separated by an electrically insulating but ionic permeable separator. Charge enters and leaves the device through current collectors on either end of the device and is transferred within the device by ions in the electrolyte.

The PB model that will be studied was originally proposed in [15] and then reformulated in a more efficient manner in [8]. Definitions of the model symbols are given in Table I. In the electrodes, the model is described by three partial differential algebraic equations

$$
\begin{gathered}
a C \frac{\partial\left(\phi_{1}-\phi_{2}\right)}{\partial t}=\sigma \frac{\partial^{2} \phi_{1}}{\partial x^{2}} \\
\epsilon \frac{\partial c}{\partial t}=D \frac{\partial^{2} c}{\partial x^{2}}-\frac{a C}{F}\left(t_{-} \frac{d q_{+}}{d q}+t_{+} \frac{d q_{-}}{d q}\right) \frac{\partial\left(\phi_{1}-\phi_{2}\right)}{\partial t} \\
\kappa\left(\frac{R T\left(t_{+}-t_{-}\right)}{F}\right) \frac{\partial}{\partial x} \ln (c)+\sigma \frac{\partial\left(\phi_{1}-\phi_{2}\right)}{\partial x} \\
+\left(\kappa \frac{\partial}{\partial x}+\sigma \frac{\partial}{\partial x}\right) \phi_{2}+i=0 .
\end{gathered}
$$

Each of these equations describe physical phenomena: charge conservation across the double layer (1a); electrolyte diffusion (1b) and a nonlinear concentration dependent version of Ohms Law (1c). In the separator, the double layer does not exist, so all terms of (1) that include $\phi_{1}-\phi_{2}$ are neglected. The model output is the voltage

$$
y=V=\left.\phi_{1}\right|_{x=L}-\left.\phi_{1}\right|_{x=0},
$$

and the model boundary conditions are detailed in [15]. The electrode is assumed to be homogeneous and so is modelled as 1D in space $x$. This means the model input is the current density $i=I / S_{A}$ where $S_{A}$ is surface area and $I$ is the current flowing across the electrodes. The model assumptions are detailed in [15].

As far as the authors are aware, no analytical solutions to the coupled, nonlinear, multi-domain, partial differential algebraic equation system (1) exists and so, to make the system tractable, spatial discretisation is implemented on (1) using the spectral collocation method as outlined in [8]. Discretisation approximates the infinite dimensional PDE problem by a finite dimensional ODE problem where the solution is obtained on grid points. This discretised system can be converted to an ODE system by solving the algebraic equation (1c) for the algebraic variable $\phi_{2}$. A solution of this equation exists so long as a reference potential has been set, for example $\phi_{1}=\left.0\right|_{x=0}$.

The performance of the $\mathrm{PB}$ model is compared to that of an EC. A summary of several popular equivalent circuit models is detailed in [17]. The "dynamic" circuit 
from [17] described by

$$
\begin{aligned}
{\left[\begin{array}{l}
\dot{x}_{1} \\
\dot{x}_{2} \\
\dot{x}_{3}
\end{array}\right] } & =\left[\begin{array}{cccc}
0 & 0 & 0 & 0 \\
0 & -\frac{1}{R_{1} C_{1}} & 0 \\
0 & 0 & -\frac{1}{R_{2} C_{2}}
\end{array}\right]\left[\begin{array}{l}
x_{1} \\
x_{2} \\
x_{3}
\end{array}\right]+\left[\begin{array}{c}
\frac{1}{C} \\
\frac{1}{C_{1}} \\
\frac{1}{C_{2}}
\end{array}\right] i \\
V & =x_{1}+x_{2}+x_{3}+R_{s} i
\end{aligned}
$$

is used.

\section{ExPERIMENTAL SETUP}

Data were obtained on a commercially available supercapacitor produced by Maxwell with part number BCA P0100 P270T01. Testing was carried out on the Biologic SP-150 machine in the Energy and Power Group, Dept of Engineering Science, Univ of Oxford. The sampling time of the data logger was 0.01s and the machine could supply a maximum current of $400 \mathrm{~mA}$. Data were obtained both in the frequency domain with electrical impedance spectroscopy (EIS) [12] which shows the magnitude and phase of the impedance function $G(j \omega)$ at open cell voltages $0.5 \mathrm{~V}, 1.5 \mathrm{~V}$ and $2.5 \mathrm{~V}$ and in the time domain. The charging profile of the time domain data is composed of square waves of irregular frequency as shown in Figure 1a. This profile was chosen to introduce excitation frequencies into the supercapacitor in a manner which was convenient with regards to the testing equipment since these machines are orientated towards constant current charges/discharges.

\section{Parameter Estimation}

Parameter estimation was carried out by considering the model as a grey box model. Estimates of the model parameters were obtained using the Matlab 'Systems Identification Toolbox' by solving the optimisation problem

$$
\begin{array}{r}
\theta^{*}=\arg \min _{\theta}\|y-\hat{y}(\theta)\|_{2} \\
\text { subject to } \theta \in \Theta \subset \mathcal{R}^{n}
\end{array}
$$

which minimises the norm of the error between the output from the experimental data output $y$ and the simulated output from the model $\hat{y}$ that is a function of the model parameters $\theta$ [11]. The subscripts $p b$ and $e c$ will respectively define parameters for the PB model (1) and the EC (3).

By introducing the definition of the diffusion coefficient

$$
D=\frac{2 \kappa\left(t_{+}\left(1-t_{+}\right)\right) R T}{F^{2} c_{0}}
$$

given in [15], the number of parameters required by the PB model (1) can be reduced to the following eleven: $\epsilon_{e}$, $\epsilon_{s}, \kappa_{e}, \kappa_{s}, a C, \sigma, t_{+}, L_{e}, L_{s}, S_{A}, c_{0}$ where the subscripts $e$ and $s$ respectively define parameters in the electrode and separator domains. It was found that only the parameters $\theta_{p b}=\left\{\kappa_{e}, a C, \sigma\right\}$, which were constrained to the set $\Theta_{p b}:=\left\{\theta_{p b}: \theta_{p b} \geq 0\right\}$, were identifiable from the data. To improve the accuracy of the overall estimation, only

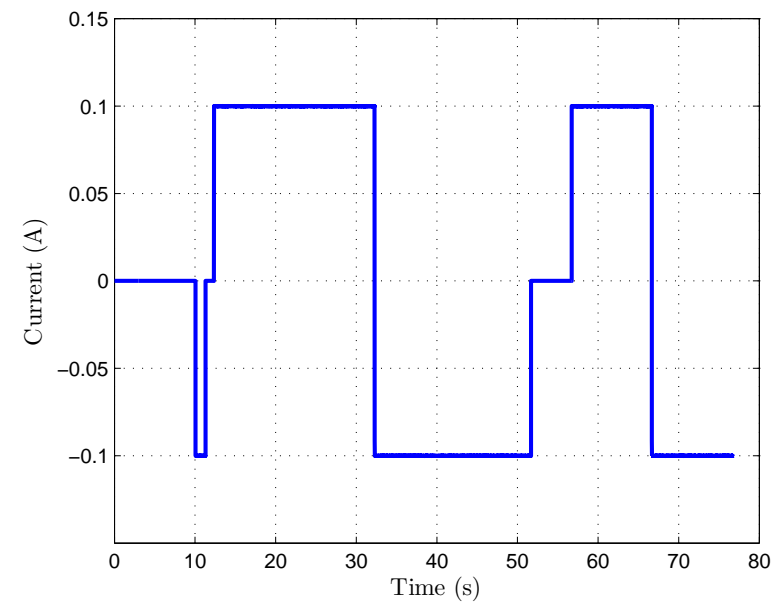

(a) Experimental square wave current charging profile.

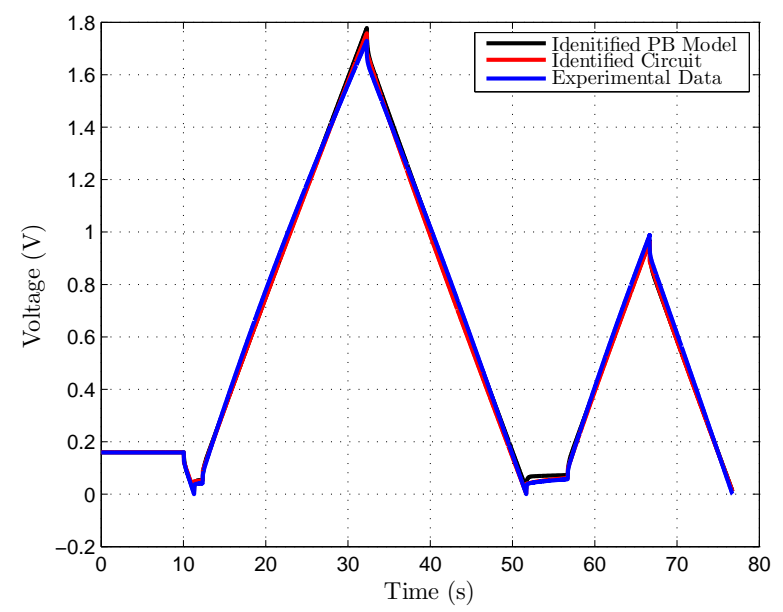

(b) Voltage responses for the EC, the PB model and experimental data.

Fig. 1: Identification in the time domain.

these parameters were estimated in the identification of the PB model, with the rest of the parameters being fixed to values given in Table IV. This is a similar approach to [13] for lithium ion batteries. Due to their physical interpretation, these fixed parameters can be measured directly. The parameters of the EC given in (3) were defined as $\theta_{e c}:=\left\{C_{1}, R_{1}, C_{2}, R_{2}, C, R_{s}\right\}$ which were constrained to be positive $\Theta_{e c}:=\left\{\theta_{e c}: \theta_{e c} \geq 0\right\}$.

The simulated results from the square wave time domain data are shown in Figure 1. It was found that the added complexity of the PB model resulted in a slightly less accurate simulation for this charging profile when compared to the EC, with the two models having error norms

$$
\begin{gathered}
\left\|y-\hat{y}_{e c}\left(\theta_{e c}\right)\right\|_{2}=1.71 \\
\left\|y-\hat{y}_{p b}\left(\theta_{p b}\right)\right\|_{2}=2.08
\end{gathered}
$$

The identified model parameters for this charging profile are shown in Tabs II \& III. 


\begin{tabular}{|c|c|c|c|c|c|}
\hline \multirow[b]{3}{*}{ Parameter } & \multicolumn{4}{|c|}{ Identified Parameters } & \multirow[b]{3}{*}{ Units } \\
\hline & \multirow[b]{2}{*}{ Time Domain } & \multicolumn{3}{|c|}{ Frequency Domain } & \\
\hline & & $0.5 \mathrm{~V} \mathrm{OCV}$ & $1.5 \mathrm{~V} \mathrm{OCV}$ & $2.5 \mathrm{~V} \mathrm{OCV}$ & \\
\hline & $5.78 \times 10^{-5}$ & $1.018 \times 10^{-4}$ & $9.89 \times 10^{-5}$ & $9.09 \times 10^{-5}$ & $\mathrm{~S} \mathrm{~m}^{-1}$ \\
\hline$a C$ & $1.91 \times 10^{5}$ & $1.08 \times 10^{6}$ & $1.27 \times 10^{6}$ & $1.35 \times 10^{6}$ & $\mathrm{~F} \mathrm{~m}^{-2}$ \\
\hline$\sigma$ & 0.002 & $4.07 \times 10^{-4}$ & $4.58 \times 10^{-4}$ & $4.84 \times 10^{-4}$ & $\mathrm{~S} \mathrm{~m}^{-1}$ \\
\hline
\end{tabular}

TABLE II: Identified parameters of the PB model.

\begin{tabular}{l|l|lll|l}
\multirow{2}{*}{} & \multicolumn{4}{|c|}{ Identified Parameters } & \\
\cline { 2 - 5 } Parameter & Time Domain & \multicolumn{3}{|c}{ Frequency Domain } & Units \\
\cline { 2 - 5 }$R_{1}$ & 1.04 & $0.5 \mathrm{~V}$ OCV & $1.5 \mathrm{~V}$ OCV & $2.5 \mathrm{~V}$ OCV & $\Omega .0018$ \\
$C_{1}$ & 13.78 & 0.142 & 0.0017 & 0.124 & $\mathrm{~F}$ \\
$R_{2}$ & 0.154 & 1.688 & 0.123 & 1.832 & $\Omega$ \\
$C_{2}$ & 0.838 & 0.156 & 0.169 & 0.194 & $\mathrm{~F}$ \\
$R_{s}$ & 0.275 & 6.695 & 7.821 & 8.299 & $\Omega$ \\
$C$ & 1.265 & 0.0987 & 0.0977 & 0.0984 & $\mathrm{~F}$
\end{tabular}

TABLE III: Identified parameters of the EC.

\begin{tabular}{c|c|c} 
Parameter & Value & Units \\
\hline$\epsilon_{e}$ & 0.67 & \\
$\epsilon_{s}$ & 0.6 & $\mathrm{~S} \mathrm{~m}^{-1}$ \\
$\kappa_{s}$ & 0.0259 & \\
$t_{+}$ & 0.55 & $\mathrm{~mol} \mathrm{~m}^{-3}$ \\
$c_{0}$ & 500 & $\mathrm{~m}^{2}$ \\
$S_{A}$ & 2 & $\mathrm{~m}$ \\
$L_{e}$ & $2.5 \times 10^{-5}$ & $\mathrm{~m}$
\end{tabular}

TABLE IV: Fixed parameters of the PB model.

The majority of existing studies on parameter estimation of batteries have fitted the parameters of ECs to EIS data and have shown that EIS varies with effects such as temperature, state-of-charge and open cell voltage (OCV) [18]. Figures 2, 3 \& 4 compare simulated EIS results of the EC and a linearised version of the PB model with experimental data for different open cell voltages. The errors introduced by the linearisation should not be too significant for the small amplitude signals of EIS since the logarithm of (1) is fairly benign when the concentration is far away from $0 \mathrm{~mol} \mathrm{~m}^{-3}$. The parameters of the fitted models are shown in Tables II, IV \& III. Both the PB model and the EC could be parameterised to give reasonably accurate representations of the EIS data, but the EC was more accurate. The accuracy of both model types was found to drop off at high frequencies which could be due to a faradaic current [12]. This loss of accuracy at high frequencies was also mentioned in [16] for circuits. The slow diffusion process of the ions is directly modelled in the PB model which suggests that this model should be more accurate at very low frequencies, below those sampled in the data. The variances of the parameter estimates obtained using the frequency domain data were significantly lower than those obtained from the time domain data. This is because the time domain identification process that assumes noise enters into the states which evolve with integrator dynamics. This problem of noise integration was mentioned in [7] with regards to observer design and can lead to instability, since an integrator does not exist in the $\mathcal{L}_{2}$ Hilbert space.

As predicted by the electrochemistry [12], the EIS response of the data changed at different open cell voltages, resulting in different model parameterisations. The identified parameters were found to vary with OCV voltage for both the EC and the PB model, suggesting that the models only describe the local dynamics. This implies that the PB model is missing some physical relationships that account for this variation, but it is noted that the addition of more physical relationships, such as temperature dependence, will result in even more complex and possibly nonlinear models.

The two models differed in their complexity, with the 3 state linear EC being simpler to analyse than the nonlinear PB model which needed a 20 state discretisation of the PDEs for suitable accuracy. However, this added complexity had powerful implications since the PB model could make predictions about the internal state of the system. This is shown in Figure 5 where the evolution of the ionic concentration during the square wave charging profile of Figure 1a is shown. Utilisation of this information could lead to the BMS reducing the effect of degradation by predicting ionic depletion and by helping designers to construct improved electrodes that account for the movement of the ions in a more meaningful way. It will also allow physically constrained (e.g. on the the concentration and potential) control of high current charging and discharging, which should allow higher performance than an EC. The optimal choice of supercapacitor model would therefore depend upon the particular application, if speed and simplicity is desired then an EC should be implemented, however, if more detail is wanted, then a PB model would be more desirable.

\section{Conclusions}

In this paper, the estimation of model parameters for a PDE supercapacitor model that is based on physical electrochemical relations was studied. The accuracy 


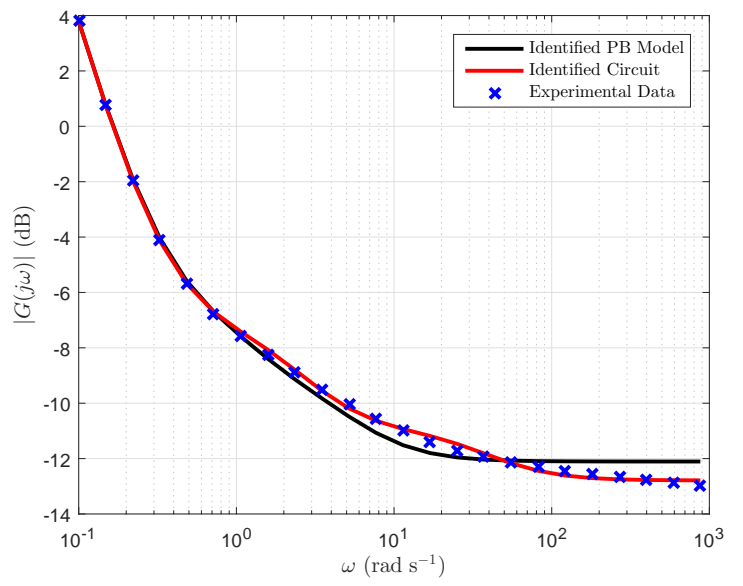

(a) Impedance function magnitude at $0.5 \mathrm{~V}$ OCV.

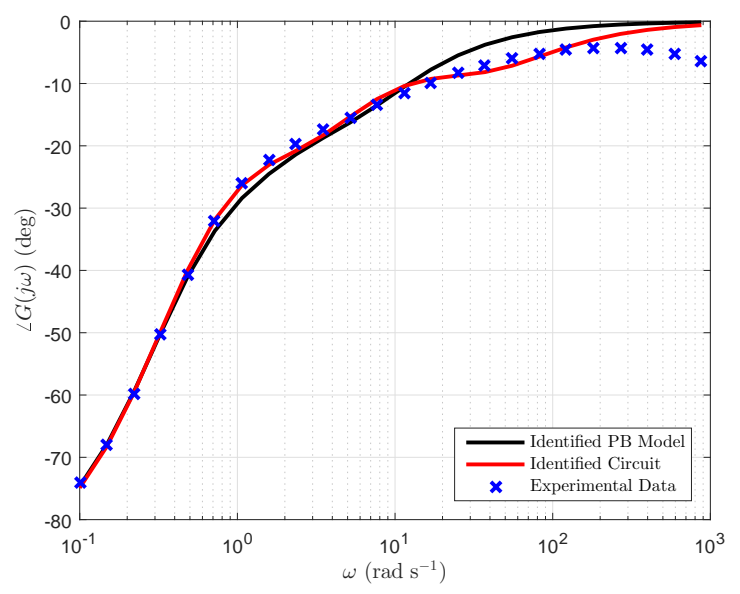

(b) Impedance function phase at $0.5 \mathrm{~V}$ OCV.

Fig. 2: EIS data at an open cell voltage of $0.5 \mathrm{~V}$

of this model was compared to that of a $2^{\text {nd }}$ order circuit. The simulated output of the identified model was shown to accurately describe experimental data both in the frequency domain with electrical impedance spectroscopy (EIS) results and in the time domain with charge/discharge current profiles composed of square waves. At different open cell voltages, the supercapacitor gave different EIS readings and this resulted in different parameterisations of the physical model and the circuit, implying they could only describe the local dynamics. The benefits and disadvantages of physical models and circuits are discussed, with the circuits being simpler while physical models are more descriptive and enable predictions to be made about the internal state of the supercapacitor, not simply its input/output response.

\section{ACKnowledgments}

The authors appreciate the work of Ben Janoff who obtained the supercapacitor experimental data and Dr Alan Ruddell, STFC Rutherford Appleton Laboratory, for supplying the testing equipment.

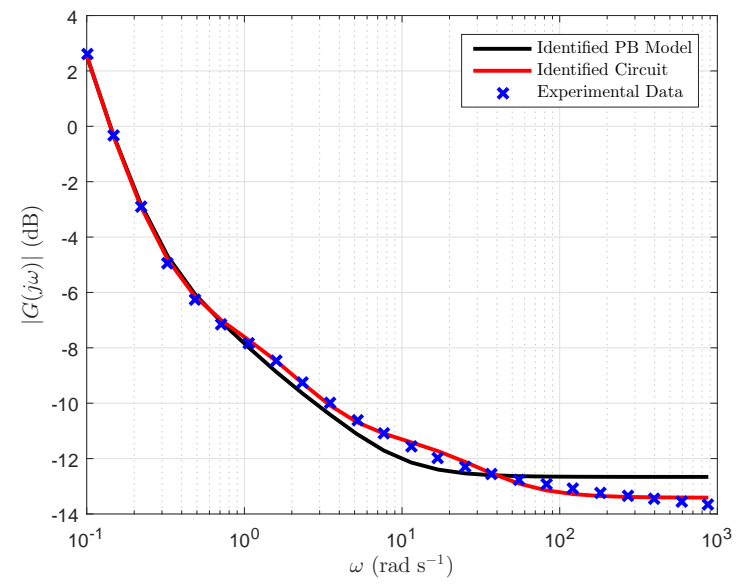

(a) Impedance function magnitude at $1.5 \mathrm{~V}$ OCV.

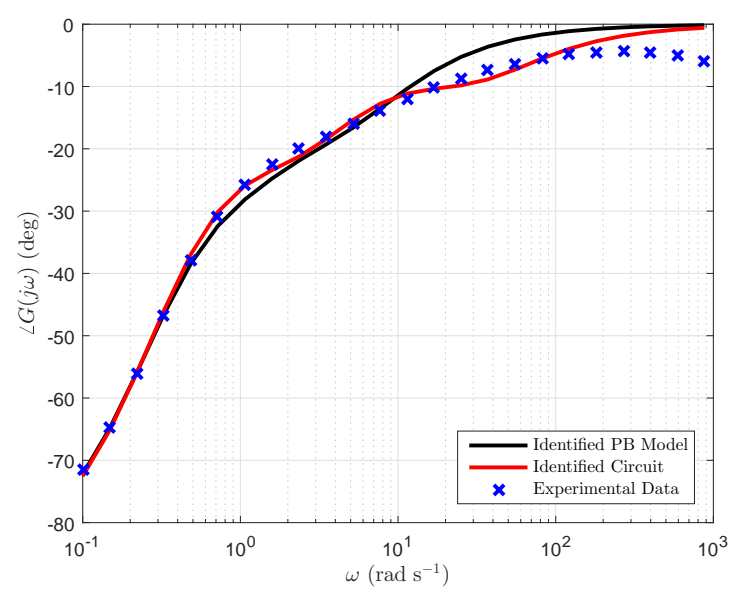

(b) Impedance function phase at $1.5 \mathrm{~V}$ OCV.

Fig. 3: EIS data at an open cell voltage of $1.5 \mathrm{~V}$

\section{REFERENCES}

[1] S Allu, B Velamur Asokan, WA Shelton, B Philip, and S Pannala. A generalized multi-dimensional mathematical model for charging and discharging processes in a supercapacitor. Journal of Power Sources, 256(0):369 - 382, 2014.

[2] AM Bizeray, S Zhao, SR Duncan, and DA Howey. Lithium-ion battery thermal-electrochemical modelbased state estimation using orthogonal collocation and a modified extended kalman filter. Journal of Power Sources, 296:400-412, 2015.

[3] O Brune. Synthesis of a finite two-terminal network whose driving-point impedance is a prescribed function of frequency. $\mathrm{PhD}$ thesis, Massachusetts Institute of Technology, 1931.

[4] N Chaturvedi, R Klein, J Christensen, and A Ahmed, Jand Kojic. Algorithms for advanced battery-management systems. Control Systems, IEEE, 30(3):49-68, 2010.

[5] A d'Entremont and L Pilon. First-principles thermal modeling of electric double layer capacitors under 


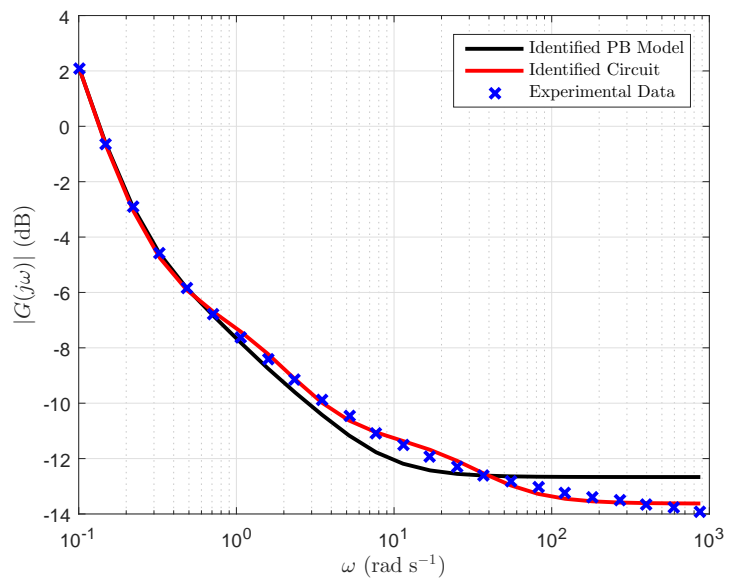

(a) Impedance function magnitude at $2.5 \mathrm{~V}$ OCV.

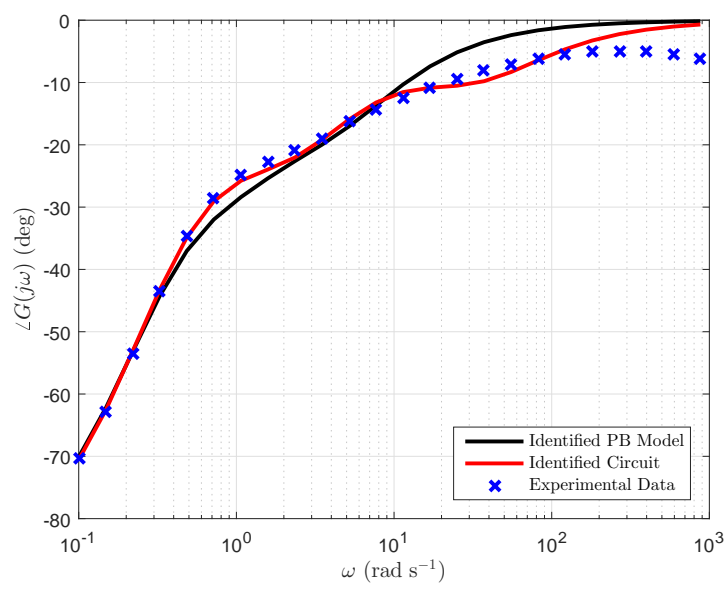

(b) Impedance function phase at $2.5 \mathrm{~V}$ OCV.

Fig. 4: EIS data at an open cell voltage of $2.5 \mathrm{~V}$

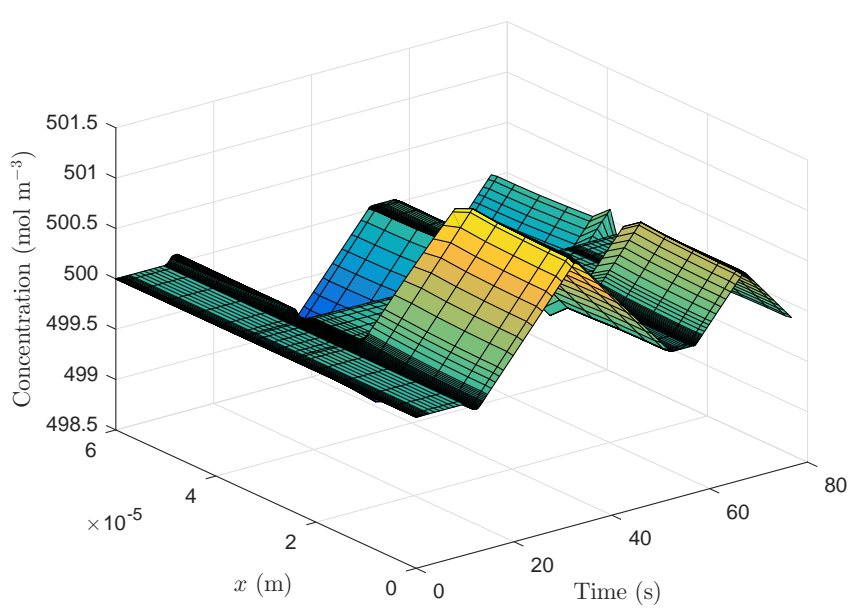

Fig. 5: The simulated diffusion of the ionic concentration during the square wave input charging profile.

constant-current cycling. Journal of Power Sources, 246:887-898, 2014.
[6] M Doyle, TF Fuller, and J Newman. Modeling of galvanostatic charge and discharge of the lithium/polymer/insertion cell. Journal of the Electrochemical Society, 140(6):1526-1533, 1993.

[7] R Drummond and SR Duncan. On observer performance for an electrochemical supercapacitor model. In 2015 IEEE Conference on Control Applications, pages 1260-1265. IEEE, 2015.

[8] R Drummond, DA Howey, and SR Duncan. Loworder mathematical modelling of electric double layer supercapacitors using spectral methods. Journal of Power Sources, 277:317-328, 2015.

[9] T Franke and JF Krems. Interacting with limited mobility resources: Psychological range levels in electric vehicle use. Transportation Research Part A: Policy and Practice, 48:109 - 122, 2013.

[10] SA Kislenko, RH Amirov, and IS Samoylov. Molecular dynamics simulation of the electrical double layer in ionic liquids. Journal of Physics: Conference Series, 418(1):12:21, 2013.

[11] L Ljung. System identification: Theory for the user. PTR Prentice Hall Information and System Sciences Series, 198, 1987.

[12] M Lu, F Beguin, and E Frackowiak. Supercapacitors: Materials, Systems and Applications. John Wiley \& Sons, 2013.

[13] AP Schmidt, M Bitzer, AW Imre, and L Guzzella. Experiment-driven electrochemical modeling and systematic parameterization for a lithium-ion battery cell. Journal of Power Sources, 195(15):5071 5080, 2010.

[14] RL Spyker and RM Nelms. Classical equivalent circuit parameters for a double-layer capacitor. Aerospace and Electronic Systems, IEEE Transactions on, 36(3):829-836, 2000.

[15] MW Verbrugge and P Liu. Microstructural analysis and mathematical modeling of electric double-layer supercapacitors. Journal of The Electrochemical Society, 152(5):D79-D87, 2005.

[16] L Zhang, X Hu, Z Wang, F Sun, and DG Dorrell. Experimental impedance investigation of an ultracapacitor at different conditions for electric vehicle applications. Journal of Power Sources, 287:129 138, 2015.

[17] L Zhang, Z Wang, X Hu, F Sun, and DG Dorrell. A comparative study of equivalent circuit models of ultracapacitors for electric vehicles. Journal of Power Sources, 274:899-906, 2015.

[18] L Zhang, Z Wang, F Sun, and DG Dorrell. Online parameter identification of ultracapacitor models using the extended Kalman filter. Energies, 7(5):3204$3217,2014$. 